\title{
Nonlocal impedances and the Casimir entropy at low temperatures
}

\author{
V. B. Svetovoy* \\ MESA + Research Institute, University of Twente, P. O. 217, 7500 AE Enschede, The Netherlands \\ R. Esquivel ${ }^{\dagger}$ \\ Instituto de Fisica, Universidad Nacional Autónoma de México, Apartado Postal 20-364, DF 01000 México, México
}

(Received 8 April 2005; published 13 September 2005)

\begin{abstract}
The problem with the temperature dependence of the Casimir force is investigated. Specifically, the entropy behavior in the low temperature limit, which caused debates in the literature, is analyzed. It is stressed that the behavior of the relaxation frequency in the $T \rightarrow 0$ limit does not play a physical role since the anomalous skin effect dominates in this range. In contrast with the previous works, where the approximate Leontovich impedance was used for analysis of nonlocal effects, we give description of the problem in terms of exact nonlocal impedances. It is found that the Casimir entropy is going to zero at $T \rightarrow 0$ only in the case when $s$ polarization does not contribute to the classical part of the Casimir force. However, the entropy approaching zero from the negative side that, in our opinion, cannot be considered as thermodynamically satisfactory. The resolution of the negative entropy problem proposed in the literature is analyzed and it is shown that it cannot be considered as complete. The crisis with the thermal Casimir effect is stressed.
\end{abstract}

DOI: 10.1103/PhysRevE.72.036113

PACS number(s): 11.10.Gh, 11.10.Wx, 42.50.Pq, 78.20.Ci

\section{INTRODUCTION}

An attractive force between uncharged metallic plates, predicted in 1948 by Casimir [1], is one of the most striking macroscopic manifestations of quantum vacuum. Recently this force became a subject of systematic experimental investigation [2-9]. The force between ideal metals at zero temperature $[1]$,

$$
F=-\frac{\pi^{2} \hbar c}{240 a^{4}}
$$

depends only on the separation $a$ and fundamental constants. In reality the force is measured at finite temperature between deposited metallic films, which have finite conductivity and roughness. Correction to Eq. (1) due to finite conductivity can be as large as $50 \%$ for small separations $a \sim 100 \mathrm{~nm}$. Contribution of the finite temperature to this correction is not large but caused a lot of controversy in the literature (see Ref. [10] for a recent review). The essence of the problem lies in the classic contribution to the Casimir force, which dominates at large distances between plates or at high temperature. Calculations made for ideal metals at finite temperature $[11,12]$ showed that $s$ - and $p$-polarized modes of electromagnetic field gave equal contributions to the force. At the same time the Lifshitz theory of fluctuating fields $[13,14]$ predicted zero contribution for $s$ polarization. For the first time the problem was recognized many years ago. For reconciliation of the results Schwinger, DeRaad, and Milton (SDM) [15] proposed a special prescription to be used with the Lifshitz formula, one must take first the limit $\varepsilon \rightarrow \infty$ for the metal permittivity and only then allow the frequency $\omega$ to

\footnotetext{
*Electronic address: v.b.svetovoy@el.utwente.nl; on leave from Yaroslavl University, Yaroslavl, Russia.

†Electronic address: raul@fisica.unam.mx
}

go to zero. Modern calculations concerned with nonideal metals were confronted with the problem again.

Different approaches to resolve the problem have been proposed in the literature, which resulted in different temperature corrections to the Casimir force. Boström and Sernelius [16] used the Lifshitz formula with the Drude dielectric function and found that $s$ polarization did not contribute in the classical limit ( $n=0$ term in the Lifshitz formula) independently on the Drude parameters. In this approach there is no continuous transition to the ideal metal case and the predicted temperature correction is in contradiction with the Lamoreaux experiment [2]. However, physically this approach is well motivated since the Drude dielectric function is working especially well at low frequencies. Bordag et al. [17] used the plasma model dielectric function, for which $\varepsilon$ at low frequencies increases faster $\left(\omega^{-2}\right)$ than for the Drude model $\left(\omega^{-1}\right)$. They found that $s$ polarization gives finite contribution in the classical limit, which coincides with the ideal metal result when the plasma frequency $\omega_{p}$ is going to infinity. The temperature correction in this approach is very close to that for the ideal metal and negligible at small separations between plates. A weak point of this approach is that no known material behaves at low frequency according to the plasma model. Svetovoy and Lokhanin [18] proposed to use SDM prescription for the $n=0$ term in the Lifshitz formula for real metals also. Later it was shown [19] that this prescription follows from very general dimensional analysis of the classical contribution to the force if one demands continuous transition to the ideal metal case. The temperature correction happened to be small but observable at small separations between bodies.

A new round of discussion has started when a thermodynamical problem connected with the Casimir free energy has been revealed [20]. The idea was to use the Nernst heat theorem as a guiding principle to choose between different approaches to the temperature correction. According to this 
theorem the entropy must go to zero in the limit of zero temperature. It was noted [20] that the Drude relaxation frequency $\omega_{\tau}$ vanishes with $T$ and, therefore, the plasma dielectric function is realized at $T \rightarrow 0$. In this case the leading term in the temperature correction is $\sim T^{3}[17]$ and the entropy is safely going to zero as $S \sim T^{2}$. Two other approaches predict the leading term in the correction $\sim T$ and finite entropy at $T=0$, positive and negative for the approaches $[18,16]$, respectively. However, the following analysis revealed that the situation is not as simple. The anomalous skin effect was shown to be important for the temperature correction at low temperatures [21]. With the use of the Leontovich impedance for the anomalous skin effect it was demonstrated that the entropy is going to zero only if SDM prescription is used for the $n=0$ term. On the other hand, it was noted [22] and expressed later more clearly [23] that any real material contains a number of defects, which are responsible for the residual resistance at $T=0$. Equivalently it means that $\omega_{\tau}$ becomes very small but finite at $T=0$. It was shown, that in this case, the entropy disappears at sufficiently low temperature $[22,23]$. Therefore, again we have a confusing situation where each approach has its own reasoning.

We would like to emphasize that at low temperatures the anomalous skin effect plays an important role and should be taken into account in any reasonable calculations. Because $\omega_{\tau}$ decreases fast with the temperature, at sufficiently low temperature inevitably the mean free path $l=v_{F} / \omega_{\tau}(T)$ for electrons becomes much larger then the field penetration depth $\delta$. When this happens the relaxation frequency does not play a physical role any more. Instead of $\omega_{\tau}$ the physical significance gets the other frequency, $\Omega=\left(v_{F} / c\right) \omega_{p}$, which is often used as a characteristic frequency of anomalous skin effect. For this reason the question, does $\omega_{\tau}$ go to zero or have some residual value at $T \rightarrow 0$, becomes unimportant. This ideology was developed in Ref. [21] in context of the thermal correction to the Casimir force. For the description of the anomalous skin effect the Leontovich impedance was used there.

The approximate Leontovich impedance was used for calculations [21,24,25] (see additional discussion in Refs. [26-28]). The approach similar to the Leontovich impedance was developed also in Refs. [29,30]. This impedance describes well the propagating electromagnetic field, but it was not clear why in the local limit it gives the result different from the dielectric function approach [21]. In Refs. [31-33] it was demonstrated that the use of the exact impedances is in agreement with the dielectric function approach. It became clear that the point of contradiction is the transverse momentum, which is neglected in the Leontovich impedance $[26,34,35]$. In our paper [36] a general approach to the nonlocal impedances was developed for applications in the Casimir force calculations. It was shown that for real metals both contributions in the force from propagating and evanescent fields are important. The propagating fields can be described well by the Leontovich impedance, but the same is not true for the evanescent fields. The latter ones should be described by more general impedances, for which dependence on the transverse momentum cannot be neglected. Explicit expressions for these impedances were presented in Ref. [36].
It is important to notice that the relevance of spatial dispersion effects depends on the separation between the slabs, being more important at short separations. For two Au slabs, at a separation of the order of the plasma wavelength of $\mathrm{Au}$ $(130-140 \mathrm{~nm})$, the difference between the local and nonlocal calculation is $0.2 \%$, and can be significant when experimental errors of the order of $0.5 \%$ are claimed [8]. For the hydrodynamic model Ref. [32] and Ref. [37] give the same results, using a dielectric function valid in a wide range of frequencies not only at the infrared as stated in Ref. [8].

Inadequacy of the Leontovich impedance forced us to reconsider the result of Ref. [21] for the entropy behavior in the low temperature limit. In this paper we calculate analytically the temperature correction to the Casimir free energy using the general approach to the nonlocal impedances [36].

The paper is organized as follows. In Sec. II we separate the temperature-dependent part of the free energy and transform it to the form convenient for calculations. In Sec. III the nonlocal impedances at low temperature are discussed. In Sec. IV we give analytic expressions for the free energy in two limit cases. The entropy behavior at $T \rightarrow 0$ and discussion are given in Sec. V. In the last section we present our conclusions.

\section{TEMPERATURE-DEPENDENT PART OF THE FREE ENERGY}

The Casimir force at nonzero temperature between plates made of real materials is given by the Lifshitz formula [14]. For the free energy $\mathcal{F}(a, T)$ this formula can be presented in the following form:

$$
\mathcal{F}(a, T)=\frac{k T}{8 \pi a^{2}} \sum_{n=0}^{\infty} \int_{\xi_{n}}^{\infty} d y y\left\{\ln \left[1-r_{s}^{2}\left(\xi_{n}, y\right) e^{-y}\right]+\left(r_{s} \rightarrow r_{p}\right)\right\},
$$

where $\xi_{n}$ are the dimensionless Matsubara frequencies defined with respect to the characteristic frequency $\omega_{a}$,

$$
\xi_{n}=\frac{\zeta_{n}}{\omega_{a}}, \quad \zeta_{n}=\frac{2 \pi k T}{\hbar} n, \quad \omega_{a}=\frac{c}{2 a}
$$

In Eq. (2) $r_{s}$ and $r_{p}$ are the reflection coefficients for $s$ and $p$ polarizations, respectively. The integration variable $y$ is defined via the physical values as

$$
y=2 a \sqrt{\zeta_{n}^{2} / c^{2}+q^{2}},
$$

where $q$ is the absolute value of the wave vector along the plate.

The problem with the thermal correction comes from the $n=0$ term in Eq. (2), which will be denoted as $\mathcal{F}_{0}(a, T)$. There is no agreement between different authors [16-18] what is the reflection coefficient $r_{s}(0, y)$ in this term. The $n$ $=0$ term describes the classical contribution to the free energy, which dominates at large separations or high temperatures. Without loss of generality it can be parametrized as 


$$
\mathcal{F}_{0}(a, T)=-\alpha \frac{k T}{8 \pi a^{2}} \zeta(3) .
$$

Here $\alpha$ is a dimensionless function of material parameters and separation $a$. This form of $\mathcal{F}_{0}$ follows from a simple dimensional analysis [19] in the classical limit (not considering the Plank constant $\hbar$ ). Different approaches to the temperature correction problem give different values of $\alpha$. This value will be kept arbitrary in the calculations and will be specified only for the discussion of the final result.

We are interested in the temperature-dependent part of the free energy, which is responsible for the entropy. To separate the temperature-independent part, let us rewrite the free energy in the following form:

$$
\mathcal{F}(a, T)=\mathcal{F}_{0}(a, T)+\frac{\hbar c}{16 \pi a^{3}} \frac{\tau}{2 \pi} \sum_{n=1}^{\infty}\left[G_{s}(n \tau)+G_{p}(n \tau)\right],
$$

where the functions $G_{i}(n \tau)(i=s, p)$ are defined as

$$
G_{i}(n \tau)=\int_{n \tau}^{\infty} d y y \ln \left[1-r_{i}^{2}(n \tau, y) e^{-y}\right]
$$

and $n \tau$ was introduced instead of $\xi_{n}$. The parameter $\tau$,

$$
\tau=\frac{2 \pi T}{T_{\text {eff }}}, \quad k T_{\text {eff }}=\frac{\hbar c}{2 a}=\hbar \omega_{a},
$$

is a dimensionless temperature. It is convenient to rewrite the sum in Eq. (6) using the Abel-Plana formula

$$
\begin{aligned}
\frac{\tau}{2 \pi} \sum_{n=1}^{\infty} G_{i}(n \tau)= & \frac{1}{2 \pi} \int_{0}^{\infty} G_{i}(x) d x+\frac{\tau}{2 \pi}\left(\frac{1}{2} G_{i}(\tau)-\int_{0}^{1} G_{i}(\tau t) d t\right. \\
& \left.-2 \operatorname{Im} \int_{0}^{\infty} \frac{G_{i}(\tau+i t \tau)}{e^{2 \pi t}-1} d t\right)
\end{aligned}
$$

The first term on the right-hand side does not depend on temperature, but all the other terms describe the temperature correction. The temperature-dependent part of the free energy $\Delta \mathcal{F}(a, T)=\mathcal{F}(a, T)-\mathcal{F}(a, 0)$ can be presented then in the following form:

$$
\begin{aligned}
\Delta \mathcal{F}(a, T)= & \mathcal{F}_{0}(a, T)+\frac{k T}{8 \pi a^{2}}\left(\frac{1}{2} G_{s}(\tau)-\int_{0}^{1} G_{s}(\tau t) d t\right. \\
& \left.-2 \operatorname{Im} \int_{0}^{\infty} \frac{G_{s}(\tau+i t \tau)}{e^{2 \pi t}-1} d t+(s \rightarrow p)\right) .
\end{aligned}
$$

It is important to see clearly which frequencies give the main contribution to $\Delta \mathcal{F}(a, T)$. Indeed, the most important contribution to the temperature-independent part comes from the Matsubara frequencies $\zeta_{n} \sim \omega_{a}$ or $n \tau \sim 1$. The same is not true for $\Delta \mathcal{F}(a, T)$. As one can see from Eq. (10) the important values of the dimensionless frequency $\xi=\tau t$ are of the order of 1 or $\zeta \sim \tau \omega_{a}$. We are analyzing the temperature behavior in the low temperature range, where $\tau \ll 1$. Therefore, frequencies much smaller than the characteristic frequency $\omega_{a}$ give the main contribution to the temperature-dependent part of the free energy.

\section{NONLOCAL IMPEDANCES AT LOW FREQUENCIES}

As was mentioned in the Introduction, at low temperatures the importance of the anomalous skin effect significantly increases. Description of this effect is given within the theory of nonlocal interaction between the electromagnetic field and a metal. In this theory, the reflectivity of the metal is described by the surface impedances. The impedances are connected with the nonlocal dielectric functions by the general relations [38]

$$
Z_{s}(\omega, q)=\frac{i}{\pi} \frac{\omega}{c} \int_{-\infty}^{\infty} \frac{d k_{z}}{\left(\omega^{2} / c^{2}\right) \varepsilon_{t}-k^{2}}
$$

$$
Z_{p}(\omega, q)=\frac{i}{\pi} \frac{\omega}{c} \int_{-\infty}^{\infty} \frac{d k_{z}}{k^{2}}\left(\frac{q^{2}}{\left(\omega^{2} / c^{2}\right) \varepsilon_{l}}+\frac{k_{z}^{2}}{\left(\omega^{2} / c^{2}\right) \varepsilon_{t}-k^{2}}\right),
$$

where $k=\sqrt{q^{2}+k_{z}^{2}}$ is the wave number, $\varepsilon_{t}(k, \omega)$ and $\varepsilon_{l}(k, \omega)$ are the nonlocal dielectric functions describing the material response to transverse and longitudinal electric fields, respectively. These equations are true independently on the particular model used for obtaining $\varepsilon_{t}$ and $\varepsilon_{l}$. The dielectric functions can be found, for example, by solving the Boltzmann kinetic equation [38]. The Boltzmann approximation is valid in the range $\omega<\omega_{p}$ and $q<k_{F}$, where $k_{F}$ is the Fermi wave number, and is appropriate for our problem.

The impedances Eq. (11) and Eq. (12) are analytic functions in the upper half of the complex frequency plane and can be written at imaginary frequencies $\omega=i \zeta$ using the analytic continuation. Explicit form of the dielectric functions along the imaginary axis [36] is

$$
\varepsilon_{l}(\zeta, v)=1+\frac{\omega_{p}^{2} f_{l}(v)}{\zeta\left(\zeta+\omega_{\tau}\right)}, \quad f_{l}(v)=\frac{3}{v^{2}} \frac{v-\arctan v}{v+\left(\omega_{\tau} / \zeta\right)(v-\arctan v)},
$$

$\varepsilon_{t}(\zeta, v)=1+\frac{\omega_{p}^{2} f_{t}(v)}{\zeta\left(\zeta+\omega_{\tau}\right)}, \quad f_{t}(v)=\frac{3}{2 v^{3}}\left[-v+\left(1+v^{2}\right) \arctan v\right]$

$$
v=v_{F} \frac{k}{\zeta+\omega_{\tau}}
$$

where $v_{F}$ is the Fermi velocity. The range of the anomalous skin effect corresponds to large values of $v$. When the Casimir force is calculated, $k$ is restricted by the condition $k$ $\geqslant q \sim 1 / 2 a$. On the other hand, the denominator in Eq. (15) is small and the condition $v \gg 1$ will be fulfilled at sufficiently low temperature. In this limit the dielectric functions behave as

$$
\varepsilon_{l}(\zeta, k)=1+3\left(\frac{\omega_{p}}{v_{F} k}\right)^{2}
$$




$$
\varepsilon_{t}(\zeta, k)=1+\frac{3 \pi}{4} \frac{\omega_{p}^{2}}{\zeta v_{F} k} .
$$

One can immediately see that the relaxation frequency falls out from the dielectric functions. The longitudinal function, $\varepsilon_{l}$, does not depend on frequency at all, but $k$ dependence describes the Thomas-Fermi screening of the longitudinal electric field. In the transverse function, $\varepsilon_{t}$, the term $v_{F} k$ plays a role of the relaxation frequency. The surface impedances corresponding to the functions (16), (17) were found in Ref. [36],

$$
\begin{gathered}
Z_{s}(\zeta, q)=\frac{\zeta}{c q} F(b), \\
Z_{p}(\zeta, q)=\frac{q^{2}}{\sqrt{3}} \frac{c v_{F}}{\zeta \omega_{p}}+\frac{\zeta}{c q} G(b),
\end{gathered}
$$

where the functions $F(b)$ and $G(b)$ are defined as

$$
\begin{gathered}
F(b)=\frac{2}{\pi} \int_{0}^{\infty} d \chi \frac{\cosh ^{2} \chi}{\cosh ^{3} \chi+b^{3}}, \quad G(b)=\frac{2}{\pi} \int_{0}^{\infty} d \chi \frac{\sinh ^{2} \chi}{\cosh ^{3} \chi+b^{3}} \\
b=\frac{1}{q}\left(\frac{3 \pi}{4} \frac{\omega_{p}^{2} \zeta}{c^{2} v_{F}}\right)^{1 / 3} .
\end{gathered}
$$

The asymptotics for large and small values of $b$ are

$$
\begin{gathered}
F(b)=1+O\left(b^{3}\right), \quad G(b)=\frac{1}{2}+O\left(b^{3}\right), b \ll 1, \\
F(b)=\frac{4}{3 \sqrt{3}} \frac{1}{b}+O\left(b^{-3}\right), \quad G(b)=\frac{4}{3 \sqrt{3}} \frac{1}{b}+O\left(b^{-3}\right), b \gg 1 .
\end{gathered}
$$

The Leontovich impedance for the strong anomalous skin effect [39] is reproduced at finite frequency in the limit $q$ $\rightarrow 0$ when $b \gg 1$,

$$
Z_{s}(0, \zeta)=Z_{p}(0, \zeta)=Z(\zeta)=\frac{4}{3 \sqrt{3}}\left(\frac{4}{3 \pi} \frac{v_{F}}{c} \frac{\zeta^{2}}{\omega_{p}^{2}}\right)^{1 / 3} .
$$

However, the most important contribution to the Casimir force give finite values of $q \sim 1 / 2 a$ and the limit $b \gg 1$ inevitably will be broken at some sufficiently low frequency (temperature). When $\zeta$ is so small that $b \ll 1$, the impedance $Z_{s}$ approaches the local limit $Z_{s}(q, \zeta)=\zeta / c q$, which does not depend on $\omega_{\tau}$. This is in contrast with the Leontovich impedance, which behaves in the local limit as $Z_{s}(\zeta)=\varepsilon(i \zeta)^{-1 / 2}$ $\rightarrow \sqrt{\zeta \omega_{\tau} / \omega_{p}^{2}}$. It clearly depends on the value of $\omega_{\tau}$. Indeed, the reason for this is the nondependence on $q$ of the Leontovich approximation. The impedance $Z_{p}$ also behaves very differently from the Leontovich impedance in the limit $b$ $\ll 1$, but in contrast with $Z_{s}$ it is significantly nonlocal. This is because for $b \ll 1$ the main contribution in $Z_{p}$ gives the first term in Eq. (19) responsible for the Thomas-Fermi screening.

Let us discuss now the temperature range where Eqs. (18) and (19) are true. The main condition is that the parameter $v$ in Eq. (15) must be large. The minimal value of the wave number is $k=q \sim 1 / 2 a$. The important frequencies contributing to the temperature-dependent part of the free energy Eq. (10) are $\zeta \sim 2 \pi k T / \hbar$. We assume that the relaxation frequency $\omega_{\tau}$ decreases with temperature faster than linearly and for this reason it can be neglected in the denominator of Eq. (15). At very low temperatures this assumption can be broken due to residual resistivity, but in this case $v$ will be certainly large. Therefore, the value of $v$ will be much larger than 1 if

$$
k T \ll \frac{\hbar \omega_{a}}{2 \pi} \frac{v_{F}}{c} .
$$

When this condition is met, the impedances Eq. (18), Eq. (19) can be used independently on the value of $b$. However, for large $b$, when the Leontovich impedance (24) can be used, the condition on the temperature is relaxed. This is because for $b \gg 1$, the wave number $k=q \cosh \chi \sim q b$ $\gg 1 / 2 a$. In this limit the condition $v \gg 1$ means

$$
k T \ll \frac{\hbar \omega_{p}}{4} \sqrt{\frac{3}{\pi}} \frac{v_{F}}{c} .
$$

This is the restriction on the temperature used in Ref. [21]. Therefore, the Leontovich impedance can be used in the temperature range $\hbar \omega_{a}\left(v_{F} / c\right) \ll 2 \pi k T \ll \sqrt{3 / 16 \pi} \hbar \omega_{p}\left(v_{F} / c\right)$. When the temperature is going down and obeys the condition $2 \pi k T \ll \hbar \omega_{a}\left(v_{F} / c\right)$, the $q$ dependence of the impedances becomes important and one must use Eqs. (18), (19), and (22) instead of Eq. (24).

\section{EVALUATION OF THE FREE ENERGY}

The temperature-dependent part of the free energy is defined in Eq. (10), where the functions $G_{s, p}$ are given by Eq. (7). The reflection coefficients $r_{s, p}$ can be expressed via the impedances as

$$
r_{s}=-\frac{Z_{s 0}-Z_{s}}{Z_{s 0}+Z_{s}}, \quad r_{p}=\frac{Z_{p 0}-Z_{p}}{Z_{p 0}+Z_{p}},
$$

where $Z_{s 0}$ and $Z_{p 0}$ are the "impedances" of the plain wave defined as the ratios of the electric and magnetic fields in the wave,

$$
Z_{s 0}=\frac{\zeta}{c k_{0}}, \quad Z_{p 0}=\frac{c k_{0}}{\zeta}, \quad k_{0}=\sqrt{\zeta^{2} / c^{2}+q^{2}} .
$$

Let us first calculate the function $G_{s}(\tau)$. Using Eq. (18) for the impedance $Z_{s}$ the reflection coefficient can be written in the following form:

$$
r_{s}=-\frac{1-\frac{y}{\sqrt{y^{2}-\tau^{2}}} F\left(A / \sqrt{y^{2}-\tau^{2}}\right)}{1+\frac{y}{\sqrt{y^{2}-\tau^{2}}} F\left(A / \sqrt{y^{2}-\tau^{2}}\right)},
$$

where we introduced the parameter $A$ similar to that in Ref. [21], which is defined as 


$$
A=\left(\frac{3 \pi}{4} \frac{c}{v_{F}} \frac{\omega_{p}^{2}}{\omega_{a}^{2}} \tau\right)^{1 / 3}
$$

In Eq. (7) near the lower integration limit $y \sim \tau$ the reflection coefficient tends to 1 because the argument of the function $F$ is in the Leontovich region $(b \gg 1)$ where $\left(y / \sqrt{y^{2}-\tau^{2}}\right) F\left(A / \sqrt{y^{2}-\tau^{2}}\right) \sim \tau / A \ll 1$. For this reason the contribution to the integral from the region nearby the lower limit will be $\sim \tau^{2}$. One can neglect this contribution changing the lower limit by zero and neglecting $\tau^{2}$ in $\sqrt{y^{2}-\tau^{2}}$. Introducing the integration variable $x=y / A$ one finds

$$
G_{s}(\tau)=A^{2} \int_{0}^{\infty} d x x \ln \left[1-r_{s}^{2}(1 / x) e^{-A x}\right]+O\left(\tau^{2}\right),
$$

where

$$
r_{s} \simeq-\frac{1-F(1 / x)}{1+F(1 / x)} .
$$

This integral can be analyzed in two limit cases $A \ll 1$ and $A \gg 1$. The former case is realized for extremely low temperatures $(T \ll 0.1 \mathrm{~K}$ for $a>100 \mathrm{~nm})$ and should be used to check the behavior of the entropy at $T \rightarrow 0$. At all realistic temperatures the latter case is realized. Let us consider this case first. At $A \gg 1$ the main contribution to the integral Eq. (31) comes from the region $x \sim 1 / A$. Then the argument of $F(1 / x)$ is large and we are in the Leontovich impedance region, where $F(1 / x) \simeq 4 x / 3 \sqrt{3}$. This situation was already described in Ref. [21] and the result can be written immediately,

$$
G_{s}(\tau)=-\zeta(3)+\frac{4}{3 \sqrt{3}} \frac{8}{A} \zeta(3)+O\left(1 / A^{2}\right), \quad A \gg 1 .
$$

Here an additional factor $4 / 3 \sqrt{3}$ in comparison with Eq. (29) of Ref. [21] takes into account different definitions of $A$ used in this paper. Two other terms in Eq. (10) can be easily calculated with the help of Eq. (33).

In the opposite limit $A \ll 1$ the situation is different. In this case, the important values of $x$ in the integral (31) are $x \sim 1$ and the Leontovich approximation is no longer valid. In this limit the exponent can be changed by 1 and the integral in Eq. (31) is just a number that can be found numerically by substituting the expression for $F(1 / x)$ from Eq. (20) into Eq. (32). The result will be the following:

$$
G_{s}(\tau)=-0.0938 A^{2}+O\left(A^{3}\right), \quad A \ll 1 .
$$

The important difference of this expression from that found in Ref. [21] [see Eq. (23) therein] is that $G_{s} \rightarrow 0$ when $A$ is going to zero instead of the finite value $G_{s} \rightarrow-\zeta(3)$. The reason for this change of the behavior is the reflection coefficient. When the Leontovich approximation is used in Eq. (32) $r_{s} \rightarrow 1$ at $A \rightarrow 0$ but the use of the exact impedance (18) gives $r_{s} \rightarrow 0$.
Now let us find the function $G_{p}(\tau)$. As in the case of $G_{s}(\tau)$ one can neglect $\tau^{2}$ in $\sqrt{y^{2}-\tau^{2}}$ and change the lower integration limit by zero. In the low frequency (temperature) range the first term in Eq. (19) dominates and the reflection coefficient can be presented as

$$
r_{p}=\frac{1-\frac{1}{\sqrt{3}} \frac{v_{F}}{c} \frac{\omega_{a}}{\omega_{p}} y}{1+\frac{1}{\sqrt{3}} \frac{v_{F}}{c} \frac{\omega_{a}}{\omega_{p}} y} \approx 1-\frac{2}{\sqrt{3}} \frac{v_{F}}{c} \frac{\omega_{a}}{\omega_{p}} y .
$$

Typically the reflection coefficient for $p$ polarization is approaching 1 in the low frequency region. Small correction in Eq. (35) appears as a nonlocal effect connected with the Thomas-Fermi screening. With this $r_{p}$ the integral in Eq. (7) is easily calculated and for $G_{p}(\tau)$ one finds

$$
G_{p}(\tau)=-\zeta(3)\left(1-\frac{8}{\sqrt{3}} \frac{v_{F}}{c} \frac{\omega_{a}}{\omega_{p}}\right) .
$$

It does not depend on $\tau$ at all and holds true in both limits of large and small $A$. The same conclusion was made in Ref. [21] but without the Thomas-Fermi correction.

Now we are able to present the final expressions for the temperature-dependent part of the free energy in the limits $A \ll 1$ and $A \gg 1$. Calculating the integrals in Eq. (10) using the functions Eq. (34), Eq. (36) and the definition of the $n$ $=0$ term in Eq. (5) one finds in the limit of small $A$,

$$
\begin{aligned}
\Delta \mathcal{F}(a, T)= & \frac{k T}{8 \pi a^{2}}\left[-\alpha \zeta(3)+\frac{1}{2} \zeta(3)\left(1-\frac{8 v_{F} \omega_{a}}{\sqrt{3} c \omega_{p}}\right)\right. \\
& \left.+0.0146 A^{2}+O\left(A^{3}\right)\right], \quad A \ll 1,
\end{aligned}
$$

where the first term, containing $\alpha$, originates from the $n=0$ term $\mathcal{F}_{0}(a, T)$. This expression is different from Eq. (28) in Ref. [21], where the Leontovich impedance was used. First, the coefficient $1 / 2(1+$ Thomas-Fermi correction $)$ in front of the $\zeta$ function shows that only $p$ polarization contributes to the $A$-independent part and, second, the $A$-dependent part behaves as $A^{2}$ instead of $A \ln A$. These changes are due to different behavior of the exact impedance Eq. (18) in comparison with the Leotovich impedance (24). On the contrary, for $A \gg 1$ the Leontovich impedance is a good approximation and we successfully reproduce Eq. (33) of Ref. [21],

$$
\begin{aligned}
\Delta \mathcal{F}(a, T)= & \frac{k T}{8 \pi a^{2}} \zeta(3)\left[-\alpha+1-\frac{4 v_{F} \omega_{a}}{\sqrt{3} c \omega_{p}}\right. \\
& \left.-\frac{32}{3 \sqrt{3}}\left(\frac{1-2 p_{1}}{A}\right)+O\left(A^{-2}\right)\right], \quad A \gg 1,
\end{aligned}
$$

where $p_{1}$ is a numerical coefficient the same as in Ref. [21], $p_{1}=0.0133$. The only new feature in this relation is the presence of the Thomas-Fermi correction. Note that in the case of large $A$ both polarization contribute equally to the $A$-independent term $(1 / 2+1 / 2+$ Thomas-Fermi correction). 


\section{ENTROPY AND DISCUSSION}

Before discussing the entropy behavior in the low temperature limit we should fix the parameter $\alpha$ in Eq. (5) for the $n=0$ term. Let us separate it in two parts describing $s$ and $p$ polarizations,

$$
\alpha=\alpha_{s}+\alpha_{p} .
$$

Contribution of $p$ polarization in the classical part of the free energy $\mathcal{F}_{0}(a, T)$ is not problematic. As we know the only new feature that appeared due to nonlocality is the ThomasFermi screening. It has clear physical meaning and should be present in any reasonable approach. To find $\alpha_{p}$ we must take the impedance (19) at $\zeta \rightarrow 0$ and calculate the function $-G_{p}(0) / 2$ [see Eq. (7)]. But we already found the function $G_{p}(\tau)$, which is given by Eq. (36) and in our approximation it does not depend on $\tau$ at all, therefore,

$$
\alpha_{p}=\frac{1}{2}\left(1-\frac{8}{\sqrt{3}} \frac{v_{F}}{c} \frac{\omega_{a}}{\omega_{p}}\right) .
$$

It is important that the Thomas-Fermi correction in $\alpha_{p}$ is exactly canceled with that in the $A$-independent part of the free energy (37) or (38). Therefore, the Thomas-Fermi screening finally does not contribute to the temperaturedependent part of the free energy.

The real problem is connected with the value of $\alpha_{s}$. In Boström and Sernelius approach [16] $s$ polarization does not contribute to the $n=0$ term and $\alpha_{s}=0$. When SDM prescription is used for the $n=0$ term [18] the contribution of $s$ polarization is the same as for the ideal metal, $\alpha_{s}=1 / 2$. The plasma model prescription for the $n=0$ term [17] gives $\alpha_{s}$ $=\alpha_{s}\left(\omega_{a} / \omega_{p}\right)$ as a function of the separation, which approaching $1 / 2$ at $\omega_{a} / \omega_{p} \ll 1$. Close value of $\alpha_{s}$ gives extrapolation of the Leontovich impedance from the infrared range to zero frequency used in Ref. [25]. Different values of $\alpha_{s}$ are responsible for different temperature corrections in these approaches.

At very low temperature when $A \ll 1$ the entropy calculated from Eq. (37) is

$$
S=-\frac{\partial \Delta \mathcal{F}}{\partial T}=\frac{k}{8 \pi a^{2}}\left(\alpha_{s} \zeta(3)-\frac{5}{3} 0.0146 A^{2}\right), \quad A \ll 1 .
$$

It goes to zero at $T \rightarrow 0$ only if $\alpha_{s}=0$. In this case the entropy approaches zero from the negative side as $T^{2 / 3}$. This conclusion coincides with that made in Refs. $[22,23]$ on the basis of finite residual resistivity. The use of finite $\omega_{\tau}(0)$ was criticized in Ref. [26] (see also a recent presentation [40]) on the ground that the Nernst heat theorem was formulated for equilibrium states and any defects in the material responsible for the residual resistivity should be considered as deviation from equilibrium. The objection is reasonable but here we showed that the residual resistivity did not play a physical role at low temperatures. Instead the nonlocal effects are responsible for the effective relaxation frequency [see Eq. (17)] $v_{F} k \sim v_{F} / a \sim 10^{13} \mathrm{rad} / \mathrm{s}$, which is much more important than tiny $\omega_{\tau}(0)$. Nevertheless, as Eq. (41) demonstrates the final conclusion of Refs. [22,23] holds true.
At higher temperatures when $A \gg 1$ but $\tau \ll 1$ the entropy is still negative. In this range from Eq. (38) one finds for the entropy

$$
S=\frac{k}{8 \pi a^{2}} \zeta(3)\left[\alpha_{s}-\frac{1}{2}+\frac{64}{9 \sqrt{3}}\left(\frac{1-2 p_{1}}{A}\right)\right], \quad A \gg 1,
$$

which is obviously negative for $\alpha_{s}=0$.

The entropy is a positively defined physical value and the negative value for the Casimir entropy is puzzling. Recently [35] some arguments were provided justifying the negative Casimir entropy as long as the total entropy is positive. The free energy of the whole system consists of two contributions. The main additive part comes from the short-range atomic interaction. The long-range interaction realized via fluctuating fields gives much smaller contribution to the free energy, but this contribution can be separated due to its nonadditive character (see discussion of this problem in Ref. [14]). The additive and nonadditive parts are independent on each other because the first is defined by the volume but the second depends on the separation between bodies. Usually it is assumed that the nonadditive part is given by the Casimir free energy of fluctuating fields. The idea proposed in Ref. [35] is that part of the nonadditive free energy can belong to the bodies. In this case one can write

$$
\Delta \mathcal{F}(a, T)=\Delta \mathcal{F}_{\text {body }}(a, T)+\Delta \mathcal{F}_{\text {field }}(a, T)
$$

Both terms give contribution to the entropy

$$
S(a, T)=-\frac{\partial \Delta \mathcal{F}_{\text {body }}}{\partial T}-\frac{\partial \Delta \mathcal{F}_{\text {field }}}{\partial T} .
$$

The second term here is negative at low $T$ but the first term could provide the total entropy to be positive. This idea can be true but we would like to stress that the term $\Delta \mathcal{F}_{\text {body }}(a, T)$ should be explicitly specified. This is because it gives contribution not only to the entropy, but also to the force according to the relation

$$
F(a, T)=-\frac{\partial \Delta \mathcal{F}_{\text {body }}}{\partial a}-\frac{\partial \Delta \mathcal{F}_{\text {field }}}{\partial a} .
$$

At the moment we do not know any corrections to the $\mathrm{Ca}$ simir force which appear not from the fluctuating field but from the nonadditive free energy of the bodies. In our opinion the negative Casimir entropy is the evidence of a thermodynamic problem. We should stress, however, that all the other approaches to the temperature correction equally suffer the thermodynamic problem because for $\alpha_{s} \neq 0$ the entropy is finite at $T=0$.

On the other hand, the zero contribution of $s$ polarization to the $n=0$ term has solid physical grounds. In the local case the $1 / \omega$ behavior of the dielectric function, responsible for the vanishing of the reflection coefficient $r_{s}$, is the direct result of the Ohm's law. Any attempts to change this behavior will break this law. The plasma model describes well the infrared optics but this is only an approximation, which cannot be used as a low frequency limit as was proposed in Ref. [17]. Otherwise any real metal would be a perfect conductor. 
The same is true for the impedance approach, which is extrapolated from the infrared optics to zero frequency $[25,26]$, and for the SDM prescription as in Ref. [18]. Our nonlocal analysis does not bring anything new in the $n=0$ term because in the zero frequency limit the impedance Eq. (18) coincides with the exact local impedance. It is known that properly defined local impedances reproduce the force in the dielectric function approach [32], therefore, $\alpha_{s}=0$.

There is a very simple physical explanation why $s$ polarization should not contribute to the force in the low frequency limit. If $z$ is the normal direction to the metal surface, then $s$-polarized field can be chosen as having the following nonzero components of magnetic and electric fields: $H_{x}, H_{z}$, and $E_{y}$. When $\omega \rightarrow 0$ the magnetic field can be found from the Maxwell equation $\boldsymbol{\nabla} \times \mathbf{H}=4 \pi \mathbf{j} / c$, where $\mathbf{j}$ is the external current density responsible for the fluctuating fields [14]. The electric field, which is described by the equation $\boldsymbol{\nabla} \times \mathbf{E}$ $=i \omega \mathbf{H} / c$, will be suppressed in comparison with $\mathbf{H}$ because $\omega$ is small. So in the limit $\omega \rightarrow 0 s$-polarized field degenerates to pure magnetic field. But the magnetic field penetrates freely via nonmagnetic metals that means that the reflection coefficient is going to zero. Similarly the $p$-polarized field degenerates to pure electric field in the $\omega \rightarrow 0$ limit. The electric field is screened by the metal and the reflection coefficient is 1 .

We came to a contradictory situation. From electrodynamics it follows that $\alpha_{s}=0$. On the other hand, thermodynamics shows that the Casimir entropy in this case is negative and something must be wrong. All the other approaches proposed in the literature are equally unsuccessful thermodynamically $(S \neq 0$ at $T=0)$ but, in addition, they do not follow from electrodynamics. We cannot resolve the thermodynamical problem by breaking the laws of electrodynamics. Specifically we should stress that the approach based on extrapolation of the Leontovich impedance from infrared to zero frequency [26] cannot be accepted as physical. It disregards $q$ dependence of the impedances, which plays a crucial role for evanescent field configurations. The authors postulated that the evanescent fields have the same reflection coefficients as the propagating fields. The Casimir effect is not the only physical phenomenon where the evanescent fields can be probed. In the well investigated domains like near field optics or near field microwaves $q$ dependence plays a principal role. No deviations from the standard electrodynamics were noted so far.

To all appearance the experimental situation is not in favor of $\alpha_{s}=0$. This case contradicts the Lamoreaux experiment [2]. Also there were claims that $\alpha_{s}=0$ does not agree with the experiments by Decca et al. [7]. However, very high roughness of metallic films in these experiments did not allow these claims to be considered seriously. Recently [8] the same group refined their measurements reducing the surface roughness and increasing precision of determination of the absolute separation. It is important that an experimental error of $0.6 \%$ holds in a wide range of separations from $170 \mathrm{~nm}$ to $300 \mathrm{~nm}$. However, in this experiment no attempt was made to characterize the used gold films optically. Instead, the handbook [41] optical data were used for calculation of the force. It was demonstrated that $[42,43]$ the optical data for gold films prepared in different conditions can variate very significantly. Prediction of the force with the precision better than $2 \%$ should include direct measurement of the optical properties of the films especially in the mid-infrared range [43]. Nevertheless, even with the use of the handbook optical data one can conclude that the case $\alpha_{s}=0$, probably, is not supported by the experiment. This is because the handbook optical data present the best samples. The unannealed films used in the experiment should have smaller reflection coefficients than the handbook data predict. As the result, the theoretical force was overestimated in Ref. [8]. It means that the difference between the measured force and predicted one in the case $\alpha_{s}=0$ can be only larger. Of course, this is the result of only one group and one must wait for independent confirmation of it. It should be mentioned also that the best way to see the temperature correction [35] is the change of the temperature in the experiment.

All the discussion above shows that the situation with the thermal correction to the Casimir force is in deep crisis. At present, we do not know of any approach which is in agreement with both electrodynamics and thermodynamics.

\section{CONCLUSIONS}

We analyzed behavior of the Casimir free energy at low temperatures. The main contribution to the temperaturedependent part of the free energy $\Delta \mathcal{F}$ is defined by the low frequencies $\zeta \sim 2 \pi k T / \hbar$ that is in contrast with the temperature-independent part, which is defined by the characteristic frequency $\zeta \sim \omega_{a}$. With the temperature decrease the anomalous skin effect becomes increasingly important for $\Delta \mathcal{F}$. General theory of nonlocal impedances was used for calculations. It was demonstrated that at low temperatures the relaxation frequency does not play any physical role. Instead, the physical significance obtain the frequency $v_{F} k$, where $k$ is the wave number. The approximate Leontovich impedance describe the situation well if $T \gg\left(v_{F} / c\right)\left(\hbar \omega_{a} / 2 \pi\right)$. When this condition is not satisfied one cannot use the approximate Leontovich impedance any more.

The troubling $n=0$ term in the Lifshitz formula was parametrized by the parameter $\alpha$ [see Eq. (5)], which is different for different approaches to the temperature correction discussed in the literature. This parameter was kept arbitrary in calculations. In the temperature range $\hbar \omega_{a}\left(v_{F} / c\right) \ll 2 \pi k T$ $\ll \hbar \omega_{p}\left(v_{F} / c\right)$ we reproduced for the free energy the same result as in Ref. [21], where the Leontovich impedance of the anomalous skin effect was used. However, at smaller temperatures, $2 \pi k T \ll \hbar \omega_{a}\left(v_{F} / c\right)$, the behavior of $\Delta \mathcal{F}$ drastically changes because dependence of the impedance $Z_{s}$ on the transverse momentum $q$ becomes important. It was demonstrated that the entropy is going to zero in the limit $T \rightarrow 0$ only in the case when $s$ polarization does not contribute to the $n=0$ term $\left(\alpha_{s}=0\right)$. In all other cases the entropy is finite at $T=0$.

However, even in the case $\alpha_{s}=0$ the entropy at low temperatures is negative that, in our opinion, indicates the presence of the thermodynamic problem. It was demonstrated that the idea on total positive entropy proposed in Ref. [35] 
is at least incomplete. We concluded that the thermal Casimir force is in deep crisis and any approach to resolve the problem should respect both the laws of thermodynamics and electrodynamics.

Note added in proof: Recently, we became aware of the work by Bo Sernelius, Phys. Rev. B (to be published) who also analyzed the nonlocal effects in the Casimir problem.
The conclusion on the entropy behavior coincides with ours but the method of analysis is different.

\section{ACKNOWLEDGMENTS}

The authors thank Rubén Barrera for helpful discussions. Partial support from CONACyT Grant No. 44306 and DGAPA-UNAM Grant No. IN101605.
[1] H. B. G. Casimir, Proc. K. Ned. Akad. Wet. 51, 793 (1948).

[2] S. K. Lamoreaux, Phys. Rev. Lett. 78, 5 (1997); 81, 5475 (1998).

[3] U. Mohideen and A. Roy, Phys. Rev. Lett. 81, 4549 (1998); A. Roy, C.-Y. Lin, and U. Mohideen, Phys. Rev. D 60, 111101(R) (1999); B. W. Harris, F. Chen, and U. Mohideen, Phys. Rev. A 62, 052109 (2000).

[4] T. Ederth, Phys. Rev. A 62, 062104 (2000).

[5] H. B. Chan, V. A. Aksyuk, R. N. Kleiman, D. J. Bishop, and F. Capasso, Science 291, 1941 (2001); Phys. Rev. Lett. 87, 211801 (2001).

[6] G. Bressi, G. Carugno, R. Onofrio, and G. Ruoso, Phys. Rev. Lett. 88, 041804 (2002).

[7] R. S. Decca, D. López, E. Fischbach, and D. E. Krause, Phys. Rev. Lett. 91, 050402 (2003); R. S. Decca, E. Fischbach, G. L. Klimchitskaya, D. E. Krause, D. López, and V. M. Mostepanenko, Phys. Rev. D 68, 116003 (2003).

[8] S. Decca, D. López, E. Fischbach, G. L. Klimchitskaya, D. E. Krause, and V. M. Mostepanenko, Ann. Phys. (N.Y.) 318, 37 (2005).

[9] D. Iannuzzi, M. Lisanti, and F. Capasso, Proc. Natl. Acad. Sci. U.S.A. 101, 4019 (2004); M. Lissanti, D. Iannuzzi, and F. Capasso, ibid. 102, 11989 (2005);

[10] K. A. Milton, J. Phys. A 37, R209 (2004).

[11] J. Mehra, Physica (Amsterdam) 37, 145 (1967).

[12] L. S. Brown and G. J. Maclay, Phys. Rev. 184, 1272 (1969).

[13] E. M. Lifshitz, Zh. Eksp. Teor. Fiz. 29, 94 (1956) [Sov. Phys. JETP 2, 73 (1956)].

[14] E. M. Lifshitz and L. P. Pitaevskii, Statistical Physics, Part 2 (Pergamon, Oxford, 1980).

[15] J. Schwinger, L. L. DeRaad, and K. A. Milton, Ann. Phys. (N.Y.) 115, 1 (1978).

[16] M. Boström and B. E. Sernelius, Phys. Rev. Lett. 84, 4757 (2000).

[17] M. Bordag, B. Geyer, G. L. Klimchitskaya, and V. M. Mostepanenko, Phys. Rev. Lett. 85, 503 (2000).

[18] V. B. Svetovoy and M. V. Lokhanin, Mod. Phys. Lett. A 15, 1013 (2000); 15, 1437 (2000).

[19] V. B. Svetovoy and M. V. Lokhanin, Phys. Lett. A 280, 177 (2001).

[20] V. B. Bezerra, G. L. Klimchitskaya, and V. M. Mostepanenko, Phys. Rev. A 65, 052113 (2002).

[21] V. B. Svetovoy and M. V. Lokhanin, Phys. Rev. A 67, 022113
(2003).

[22] J. S. Høye, I. Brevik, J. B. Aarseth, and K. A. Milton, Phys. Rev. E 67, 056116 (2003).

[23] M. Boström and B. E. Sernelius, Physica A 339, 53 (2004).

[24] V. B. Bezerra, G. L. Klimchitskaya, and C. Romero, Phys. Rev. A 65, 012111 (2002).

[25] B. Geyer, G. L. Klimchitskaya, and V. M. Mostepanenko, Phys. Rev. A 67, 062102 (2003).

[26] V. B. Bezerra, G. L. Klimchitskaya, V. M. Mostepanenko, and C. Romero, Phys. Rev. A 69, 022119 (2004).

[27] V. B. Svetovoy, Phys. Rev. A 70, 016101 (2004).

[28] B. Geyer, G. L. Klimchitskaya, and V. M. Mostepanenko, Phys. Rev. A 70, 016102 (2004).

[29] J. R. Torgerson and S. K. Lamoreaux, Phys. Rev. E 70, $047102(2004)$

[30] S. K. Lamoreaux, Rep. Prog. Phys. 68, 201 (2005).

[31] W. L. Mochán, C. Villarreal, and R. Esquivel-Sirvent, Rev. Mex. Fis. 48, 339 (2002).

[32] R. Esquivel, C. Villarreal, and W. L. Mochán, Phys. Rev. A 68, 052103 (2003).

[33] R. Esquivel, C. Villarreal, and W. L. Mochán, Phys. Rev. A 71, 029904(E) (2005).

[34] I. Brevik, J. Aarseth, J. S. Høye, and K. A. Milton, in Quantum Field Theory Under the Influence of External Conditions, edited by K. A. Milton (Rinton, Princeton, NJ, 2004), p. 54.

[35] I. Brevik, J. Aarseth, J. S. Høye, and K. A. Milton, Phys. Rev. E 71, 056101 (2005).

[36] R. Esquivel, and V. B. Svetovoy, Phys. Rev. A 69, 062102 (2004).

[37] J. Heinrichs, Phys. Rev. B 11, 3625 (1975).

[38] K. L. Kliewer and R. Fuchs, Phys. Rev. 172, 607 (1968).

[39] A. A. Abrikosov, Fundamentals of the Theory of Metals (North-Holland, Amsterdam, 1988).

[40] V. B. Bezerra, R. S. Decca, E. Fischbach, B. Geyer, G. L. Klimchtskaya, D. E. Krause, D. López, V. M. Mostepanenko, and C. Romero, Int. J. Mod. Phys. A 20, 2205 (2005).

[41] Handbook of Optical Constants of Solids, edited by E. D. Palik (Academic, New York, 1995).

[42] V. B. Svetovoy, in Quantum Field Theory Under the Influence of External Conditions, edited by K. A. Milton (Rinton, Princeton, NJ, 2004), p. 76.

[43] V. B. Svetovoy, cond-mat/0412123 (unpublished). 